\title{
Uma Aplicação da Matemática Nebulosa na Usabilidade de Pacotes Estatísticos
}

\author{
Rodrigo Costa dos Santos ${ }^{1}$, Maria Augusta Soares Machado ${ }^{2}$, Danilo Jusan Santos ${ }^{3}$, \\ Rafael Tavares Carneiro de Campos ${ }^{4}$ \\ 1,2,3,4 Faculdades IBMEC-RJ, Av. Pres. Wilson, 118 - Centro, Rio de Janeiro, RJ. \\ ${ }^{1}$ rodrigo.santos@al.ibmecri.edu.br $;{ }^{2}$ mmachado@ibmecri.br $;{ }^{3,4}$ usabilidadeibmec@gmail.com
}

\begin{abstract}
Resumo
O presente trabalho apresenta uma aplicação da matemática nebulosa com a finalidade de se elaborar futuros estudos comparativos de pacotes estatísticos com relação à usabilidade. A metodologia foi aplicada ao pacote PHSTAT. Utilizou-se um questionário para a coleta da opinião dos usuários, dos quais foram obtidos os valores preliminares da usabilidade desse pacote. Para se obter cientificamente o resultado inicial dessa pesquisa, foi decidido delimitar o universo dos usuários a serem estudados. Para tal, a pesquisa foi centralizada entre os usuários-alunos do curso de graduação em Administração das Faculdades IBMEC-RJ. A metodologia aqui apresentada é inovadora, e foi desenvolvida por um grupo de estudo de vinte e cinco integrantes, no Ibmec-RJ em 2006, utilizando-se da matemática nebulosa para consolidar as informações coletadas e apresentar o resultado final para usabilidade.

Palavras-chave: Matemática Nebulosa; Usabilidade de Sistemas; Ergonomia.
\end{abstract}

\begin{abstract}
This paper presents a methodology using fuzzy sets to be used to compare the usability of statistical softwares based on usability. This methodology was applied for PHSTAT package. It was used a questionnaire to collect users opinions, of which there were obtained the preliminary values of the usability of this software. To obtain scientifically the initial result of our inquiry, we resolve to delimit the universe of the users to be studied. We centralize our investigation in the users-students from the business administration course of the IBMEC-RJ. We use an innovator methodology that has been developed by a group of study of twenty five integrants, at Ibmec-RJ during 2006, using the fuzzy logic to process the information collected and presents a final result for usability.

Key-words: Fuzzy Logic; System Usability; Ergonomics.
\end{abstract}

\section{Introdução}

A necessidade de qualidade na informação torna as ferramentas de software uma parte fundamental dos sistemas de informação. As ferramentas de software constituem um grande veículo de produção de trabalhos acadêmicos e não acadêmicos.

Hoje, o Ensino e a Empresa usando as tecnologias da informação, participam no desenvolvimento de pessoas e, portanto, das nações. Todavia, devem ser consideradas suas implicações, limitações, possibilidades e especificidades.

O impacto do computador no Ensino e na Sociedade é iminente. É sabido que nos últimos vinte anos, o computador cada vez mais se torna parte integrante do dia a dia dos brasileiros. Mais e mais dispositivos "computadorizados" surgem diariamente, é só lembrar como eram certos aparelhos, veículos, telefones e até mesmo o próprio computador há alguns anos atrás.
O presente artigo pretende avaliar o software estatístico PHStat do ponto de vista da sua usabilidade, ou seja, da facilidade de uso, da eficiência e eficácia na realização de suas tarefas e na satisfação final que o sistema proporciona ao seu usuário. É utilizada uma técnica em que os usuários são instados a responder um questionário referente à usabilidade que será processado utilizando a matemática nebulosa.

Primeiramente é realizada uma revisão da literatura com relação à usabilidade de sistemas e matemática nebulosa, depois é apresentada a metodologia utilizada para realização da pesquisa, seguida da avaliação dos resultados, finalizando com as conclusões da pesquisa.

\section{$2 \quad$ Usabilidade de Sistemas}

Usabilidade é definida como a capacidade de um sistema oferecer a seu usuário, em um determinado 
contexto de uso, facilidade para a realização de tarefas, de maneira eficaz, eficiente e agradável [6].

A definição de usabilidade foi introduzida em 1971 através de métricas relacionadas à "facilidade de uso" somente. Porém, foram identificados vários critérios para mensurar a facilidade de uso, como por exemplo: tempo de aprendizado, número de erros e tolerância à falhas [14].

Em 1979, deu-se início a questão da usabilidade no contexto da Interação HumanoComputador (IHC), que é a área de pesquisa que estuda a comunicação entre pessoas e sistemas. Nesta época surgiu a possibilidade de mensuração da satisfação do usuário, ou seja, o foco passa a ser cada vez mais o usuário.

Os sistemas de informações passaram a apresentar interfaces mais complexas, visuais, coloridas. Novos dispositivos foram criados para interação, como o mouse, telas sensíveis ao toque, etc.

Nesse contexto, a usabilidade tem como objetivo elaborar interfaces de sistemas capazes de permitir uma interação fácil, agradável, com eficácia e eficiência. Ela deve capacitar a criação de interfaces transparentes de maneira a não dificultar o seu uso, permitindo ao usuário pleno controle do ambiente sem se tornar um obstáculo durante a interação.

A interface é um dos componentes fundamentais para estabelecer a comunicação com o usuário e permitir que ele interaja com o sistema [9]. Quando um sistema possui uma interface amigável e possibilita ao usuário utilizá-lo de forma intuitiva, a usabilidade pode se tornar um fator de motivação e ter seu usuário como um aliado, ao passo que se essa motivação não for atingida, essa situação pode-se reverter e se tornar um fator de rejeição do sistema.

Existem várias técnicas para avaliação de usabilidade de sistemas: baseadas em questionários, modelos formais, base de conhecimento, checklists, ensaios de interação ou monitoramento. Especificamente, no caso de um software de terceiro, que não seja desenvolvido por quem quer avaliar a usabilidade dele, a técnica mais aderente é a aplicação de questionário, pois ninguém melhor do que os próprios usuários para opinarem a respeito da usabilidade do sistema. Afinal de contas eles que estão em contato com o sistema no dia-a-dia.

\section{$3 \quad$ Matemática Nebulosa}

A Lógica Fuzzy ou matemática nebulosa foi criada em 1965 a partir da publicação do artigo intitulado "Fuzzy Sets" por Lofti A. Zadeh, engenheiro eletrônico e professor da Universidade da Califórnia, Berkeley [4].

Os Conjuntos nebulosos e a Lógica nebulosa fornecem a base para geração de técnicas poderosas para a solução de problemas, com uma vasta aplicabilidade, especialmente nas áreas de Engenharia de Controle e tomada de decisões [1].

A força da Lógica Nebulosa deriva da sua habilidade em inferir conclusões e gerar respostas baseadas em informações vagas, ambíguas e qualitativamente incompletas e imprecisas. Neste aspecto, os sistemas nebulosos têm a habilidade de raciocinar de forma semelhante à dos humanos. Seu comportamento é representado de maneira muito simples e natural, levando à construção de sistemas compreensíveis e de fácil manutenção [10].

A Lógica nebulosa é baseada na teoria dos Conjuntos Nebulosos. Esta é uma generalização da teoria dos Conjuntos Tradicionais para resolver os paradoxos gerados a partir da classificação "verdadeira ou falsa" da Lógica Clássica. Tradicionalmente, uma proposição lógica tem dois extremos: ou "completamente verdadeiro" ou "completamente falso". Entretanto, na Lógica nebulosa, uma premissa varia em grau de verdade de 0 a 1 , o que leva a ser parcialmente verdadeira ou parcialmente falsa. Com a incorporação do conceito de "grau de verdade", a teoria dos Conjuntos nebulosos estende a teoria dos Conjuntos Tradicionais. Os grupos são rotulados qualitativamente (usando termos lingüísticos, tais como: alto, morno, ativo, pequeno, perto, etc.) e os elementos destes conjuntos são caracterizados variando o grau de pertinência (valor que indica o grau em que um elemento pertence a um conjunto). Por exemplo, temperaturas entre $30^{\circ}$ (trinta graus) e $40^{\circ}$ (quarenta graus) pertencem ao conjunto das "temperaturas altas", embora a temperatura de $40^{\circ}$ tenha um grau de pertinência maior neste conjunto [11].

De maneira não muito bem compreendida, humanos têm a capacidade de associar um grau de pertinência a um determinado objeto sem compreender conscientemente como se chega a ele. Por exemplo, um aluno não teria dificuldade em assinalar um grau ao professor no conjunto dos "bons professores". Esse grau é alcançado imediatamente sem nenhuma análise consciente sobre os fatores que influem nessa decisão [3].

O grau de associação não é probabilidade! Basicamente é uma medida da compatibilidade do objeto com o conceito representado pelo conjunto Nebuloso. Por exemplo, o número 0,7 é a compatibilidade da temperatura de $35^{\circ}$ com a definição do conjunto nebuloso das temperaturas altas. Esse número $(0,7)$ não é a probabilidade de $35^{\circ}$ ser uma temperatura alta, pois a mesma já está definida como $35^{\circ}[3]$.

A teoria convencional de sistemas baseia-se em equações algébricas, diferenciais ou de diferença (modelos matemáticos “crisp"). Para alguns tipos de sistemas, podem ser obtidos modelos matemáticos, como por exemplo, os sistemas eletromecânicos, já que as leis físicas por trás do processo são bem entendidas e definidas. Porém, diariamente, nos deparamos com inúmeros problemas práticos, onde se torna difícil a obtenção de um nível aceitável de informações necessárias para que possamos fazer a modelagem física. Além do mais, essa tarefa é demorada e custosa. Podemos encontrar esses sistemas nas indústrias químicas e alimentícias, em Instituições Financeiras, na Biotecnologia, entre outras áreas. Uma grande parte 
desses sistemas somente pode ser obtida através do conhecimento de especialistas que participem diretamente do processo em questão. Esse conhecimento, muitas vezes pode ser vago ou impreciso para ser expresso através de modelos matemáticos [1].

\section{$4 \quad$ Metodologia}

O presente estudo apresenta características de pesquisa aplicada, pois a pesquisa aplicada tem por objetivo utilizar um caso real para fundamentar sua análise [15]. A pesquisa aplicada é motivada pela necessidade de se resolver problemas concretos com finalidade prática.

O software estatístico escolhido para ser analisado foi o PHStat. O PHStat é um software add-in para o Microsoft Excel, criado pela editora Pearson Prentice Hall para facilitar o aprendizado dos conceitos da estatística. O PHStat permite que sejam executadas várias funções de análises estatísticas em conjunto com a interface do Excel e é geralmente utilizado por usuários iniciantes no estudo da estatística [12].

Foi aplicado um questionário para avaliara usabilidade do PHStat a uma amostra de dezoito alunosusuários do segundo período dos cursos de Administração e Administração de Sistemas das faculdades Ibmec-RJ no final de 2006.

Para avaliar a usabilidade, primeiramente recorreu-se à ISO (International Organization for Standardization), que determina que a usabilidade é um dos itens considerados no tratamento de qualidade de software, através das suas normas 9126 e também pela norma 9241.

Porém, as normas da ISO não trazem um conjunto de critérios ou métricas para avaliação de usabilidade de sistemas. Por isso, as métricas utilizadas nesse estudo serão aquelas apresentadas por [13], que estipulou um conjunto de seis métricas para avaliação de usabilidade de sistema baseado numa revisão da literatura de bases de dados científicas brasileiras (revistas e anais de congressos) entre 1995 a 2006. Essas métricas de usabilidade de sistemas estão baseadas na ISO 9126 e nos critérios de avaliação segundo alguns autores como Shackel, Nielsen, Bastien \& Scapin, Jordan, Shneiderman e Quesenbery.

Segundo [13], as métricas consideradas para avaliação de usabilidade, e as que serão utilizadas por esta pesquisa são:

$\begin{array}{ll}\text { - } & \text { Facilidade de aprender } \\ \text { - } & \text { Facilidade de relembrar } \\ \text { - } & \text { Controle de erros } \\ \text { - } & \text { Eficiência } \\ \text { - } & \text { Eficácia } \\ & \text { Satisfação }\end{array}$

A facilidade de aprender ou inteligibilidade, segundo a ISO [5], é a capacidade de o software possibilitar ao usuário aprender a manuseá-lo.

Essa métrica está sendo avaliada pelos seguintes construtos:
A) Facilidade que o usuário tem ao completar uma tarefa pela primeira vez;

B) Primeira impressão que o usuário tem ao utilizar o sistema;

C) Número de tentativas realizadas para aprender concluir uma tarefa;

D) Tempo para conseguir aprender a realizar uma tarefa com sucesso;

E) Facilidade de aprender uma tarefa;

F) Número de possibilidades diferentes que o sistema oferece para realizar a mesma tarefa, por exemplo: caminho padrão versus teclas de atalhos, caminhos mais curtos, macros, botões específicos, etc;

G) Ganho de produtividade com relação à maneira mais rápida que o usuário consegue realizar uma tarefa, comparando com a maneira padrão que o sistema oferece por padrão;

H) Flexibilidade que o sistema tem para executar as tarefas de maneiras diferentes, como por exemplo: personalização de atalhos, valores, menus, macros, etc;

I) Capacidade de o sistema guiar através de sua execução com dicas, ajuda, avisos, etc;

J) Rapidez para completar uma tarefa com sucesso no sistema pela primeira vez.

A facilidade de relembrar, segundo [8], avalia as funcionalidades do sistema para que sejam fáceis de relembrar, mesmo após o usuário ficar certo período de tempo sem usá-lo, sem necessidade de um novo treinamento.

Essa métrica está sendo avaliada pelos seguintes construtos:

A) Relembrar como executar uma tarefa após um período de tempo sem utilizar o sistema;

B) Facilidades para relembrar a utilização do sistema;

C) Agilidade para relembrar o uso do sistema após um período de tempo sem utilizar o mesmo.

O controle de erros, ou operacionalidade, segundo a ISO [5], é a capacidade de o software possibilitar ao usuário operá-lo e controlá-lo.

Essa métrica está sendo avaliada pelos seguintes construtos:

A) Quantidade de erros provocados pelo sistema;

B) Tempo de retomada ao funcionamento normal do sistema quando um erro ocorre;

C) Sentimento com relação à quantidade de erros provocados pelo sistema;

D) Retrabalho devido à quantidade de erros provocados pelo sistema que causa alguma perda de informação;

E) Tempo gasto para retomar a execução da tarefa no ponto em que se parou quando um erro ocorre;

F) Satisfação com relação à recuperação do erro por parte do sistema, desfazer, refazer, voltar, salvar entes de fechar, etc.;

G) Clareza das mensagens de erros apresentadas pelo sistema; 
A eficiência, segundo a ISO [5], é a capacidade de o software possibilitar ao usuário operá-lo e controlálo.

Essa métrica está sendo avaliada pelos seguintes construtos:

A) Performance apresentada pelo sistema;

B) Velocidade na realização das tarefas;

C) Produtividade do sistema;

D) Manter o sistema sob seu controle.

A eficácia, segundo [14], avalia como as tarefas foram exatamente concluídas, o desempenho de uma tarefa focado a um usuário ou a um ambiente específico.

Essa métrica está sendo avaliada pelos seguintes construtos:

A) Quantidade de passos para realizar uma tarefa;

B) Tempo para realizar uma tarefa qualquer no sistema;

C) Número adequado de passos para realizar uma tarefa no sistema.

A satisfação ou atratividade, segundo a ISO [5], é a capacidade do software em atrair o usuário, ser agradável.

Essa métrica está sendo avaliada pelos seguintes construtos:

A) Interação com a interface do sistema;

B) Realização das tarefas no sistema, com relação à clareza das mensagens, recuperação de erros, etc.;

C) Sentimento do usuário ao usar o sistema de maneira geral.

Após a coleta de dados e a consolidação das opiniões dos usuários, foi aplicada uma metodologia usando a matemática nebulosa, a fim de encontrar um número triangular nebuloso resultante das freqüências das opiniões dos usuários para o conjunto dos construtos que compõem a métrica avaliada.

Os números triangulares nebulosos são números nebulosos especiais, que apresentam duas características muito importantes: MODA e AMPLITUDE. A Moda representa o valor do número nebuloso cuja pertinência é igual a 1 (um). A amplitude é a metade da base do número nebuloso e representa o intervalo de confiança do número. A Amplitude é inversamente proporcional à confiança que se tem no valor da função de pertinência: Quanto menor amplitude, maior a confiança nos dados; quando maior a amplitude, menor a confiança nos dados [2].

Adotou-se a escala de Likert para a resposta de cada questão. Esta é uma escala onde os respondentes são solicitados não só a concordarem ou discordarem das afirmações, mas também a informarem qual o seu grau de concordância ou discordância [7]. O tamanho da escala de Likert utilizada para medir a usabilidade foi a de cinco pontos: 0-Muito Baixa, 2-Baixa, 4-Média, 6Alta e 8-Muito Alta.

\section{$5 \quad$ Resultados}

A seguir apresentam-se as estatísticas das opiniões respondidas no questionário preenchido pelos dezoito usuários, bem como a descrição da estatística básica e em seguida os números triangulares nebulosos resultantes para cada métrica e sua interpretação.

\subsection{Descrição estatística da amostra}

Para a métrica facilidade de aprender, conforme Tabela 1, que apresenta para cada construto a freqüência da opinião dos usuários para cada valor da escala (Muito Baixa até Muito Alta), considerando que esse sistema é usado para o ensino da estatística para iniciantes, observa-se que a interface do sistema apresenta uma discreta facilidade de aprendizagem.

\begin{tabular}{|c|c|c|c|c|c|}
\hline Construto & $\mathbf{0}$ & $\mathbf{2}$ & $\mathbf{4}$ & $\mathbf{6}$ & $\mathbf{8}$ \\
\hline $\mathbf{A}$ & $0 \%$ & $33 \%$ & $33 \%$ & $17 \%$ & $17 \%$ \\
\hline $\mathbf{B}$ & $0 \%$ & $28 \%$ & $33 \%$ & $28 \%$ & $11 \%$ \\
\hline $\mathbf{C}$ & $0 \%$ & $17 \%$ & $44 \%$ & $28 \%$ & $11 \%$ \\
\hline $\mathbf{D}$ & $0 \%$ & $17 \%$ & $33 \%$ & $28 \%$ & $22 \%$ \\
\hline $\mathbf{E}$ & $0 \%$ & $33 \%$ & $22 \%$ & $45 \%$ & $0 \%$ \\
\hline $\mathbf{F}$ & $0 \%$ & $11 \%$ & $39 \%$ & $44 \%$ & $6 \%$ \\
\hline $\mathbf{G}$ & $0 \%$ & $11 \%$ & $39 \%$ & $33 \%$ & $17 \%$ \\
\hline $\mathbf{H}$ & $6 \%$ & $38 \%$ & $28 \%$ & $22 \%$ & $6 \%$ \\
\hline $\mathbf{I}$ & $0 \%$ & $22 \%$ & $22 \%$ & $56 \%$ & $0 \%$ \\
\hline $\mathbf{J}$ & $0 \%$ & $44 \%$ & $22 \%$ & $28 \%$ & $6 \%$ \\
\hline
\end{tabular}

Tabela 1 - Resultados da amostra para a métrica facilidade de aprender.

Para a métrica facilidade de relembrar, conforme Tabela 2, observando-se o resultado isoladamente, os usuários apresentam uma certa facilidade em relembrar as ações executadas pelo software, mas ainda é muito pouca.

\begin{tabular}{|c|c|c|c|c|c|}
\hline Construto & $\mathbf{0}$ & $\mathbf{2}$ & $\mathbf{4}$ & $\mathbf{6}$ & $\mathbf{8}$ \\
\hline $\mathbf{A}$ & $6 \%$ & $28 \%$ & $28 \%$ & $32 \%$ & $6 \%$ \\
\hline $\mathbf{B}$ & $0 \%$ & $6 \%$ & $33 \%$ & $44 \%$ & $17 \%$ \\
\hline $\mathbf{C}$ & $6 \%$ & $17 \%$ & $33 \%$ & $33 \%$ & $11 \%$ \\
\hline
\end{tabular}

Tabela 2 - Resultados da amostra para a métrica facilidade de relembrar.

Para a métrica controle de erros, conforme Tabela 3, aparentemente, os usuários estão medianamente satisfeitos.

Para a métrica eficiência, conforme Tabela 4, aparentemente, os usuários estão medianamente satisfeitos. Observa-se ainda uma desproporcionalidade entre os construtos analisados: o construto A tende para insatisfação total, o B tente para satisfação total e o construto $\mathrm{C}$ está exatamente em uma posição mediana. Isso mostra que, com relação à eficiência, o software não está muito coeso, variando muito em relação aos quesitos avaliados. 


\begin{tabular}{|c|c|c|c|c|c|}
\hline Construto & $\mathbf{0}$ & $\mathbf{2}$ & $\mathbf{4}$ & $\mathbf{6}$ & $\mathbf{8}$ \\
\hline $\mathbf{A}$ & $0 \%$ & $28 \%$ & $28 \%$ & $44 \%$ & $0 \%$ \\
\hline $\mathbf{B}$ & $6 \%$ & $17 \%$ & $38 \%$ & $33 \%$ & $6 \%$ \\
\hline $\mathbf{C}$ & $0 \%$ & $17 \%$ & $66 \%$ & $17 \%$ & $0 \%$ \\
\hline $\mathbf{D}$ & $0 \%$ & $39 \%$ & $50 \%$ & $11 \%$ & $0 \%$ \\
\hline $\mathbf{E}$ & $0 \%$ & $6 \%$ & $33 \%$ & $55 \%$ & $6 \%$ \\
\hline $\mathbf{F}$ & $0 \%$ & $22 \%$ & $56 \%$ & $22 \%$ & $0 \%$ \\
\hline $\mathbf{G}$ & $0 \%$ & $45 \%$ & $44 \%$ & $11 \%$ & $0 \%$ \\
\hline
\end{tabular}

Tabela 3 - Resultados da amostra para a métrica controle de erros.

\begin{tabular}{|c|c|c|c|c|c|}
\hline Construto & $\mathbf{0}$ & $\mathbf{2}$ & $\mathbf{4}$ & $\mathbf{6}$ & $\mathbf{8}$ \\
\hline $\mathbf{A}$ & $0 \%$ & $55 \%$ & $39 \%$ & $6 \%$ & $0 \%$ \\
\hline B & $0 \%$ & $6 \%$ & $17 \%$ & $60 \%$ & $17 \%$ \\
\hline $\mathbf{C}$ & $0 \%$ & $28 \%$ & $44 \%$ & $28 \%$ & $0 \%$ \\
\hline D & $0 \%$ & $6 \%$ & $66 \%$ & $28 \%$ & $0 \%$ \\
\hline
\end{tabular}

Tabela 4 - Resultados da amostra para a métrica eficiência.

Para a métrica eficácia, conforme Tabela 5, aparentemente, os usuários estão medianamente satisfeitos.

\begin{tabular}{|c|c|c|c|c|c|}
\hline Construto & $\mathbf{0}$ & $\mathbf{2}$ & $\mathbf{4}$ & $\mathbf{6}$ & $\mathbf{8}$ \\
\hline $\mathbf{A}$ & $6 \%$ & $28 \%$ & $60 \%$ & $6 \%$ & $0 \%$ \\
\hline $\mathbf{B}$ & $0 \%$ & $11 \%$ & $17 \%$ & $72 \%$ & $0 \%$ \\
\hline $\mathbf{C}$ & $0 \%$ & $28 \%$ & $66 \%$ & $6 \%$ & $0 \%$ \\
\hline
\end{tabular}

Tabela 5 - Resultados da amostra para a métrica eficácia.

Para a métrica satisfação, conforme Tabela 6, aparentemente, os usuários estão medianamente satisfeitos. Destaca-se aí uma questão importante com relação ao construto $\mathrm{B}$ que está tendendo a insatisfação total. Deve-se aumentar o tamanho da amostra antes de se afirmar que o software deve se melhorado com relação a esse quesito.

\begin{tabular}{|c|c|c|c|c|c|}
\hline Construto & $\mathbf{0}$ & $\mathbf{2}$ & $\mathbf{4}$ & $\mathbf{6}$ & $\mathbf{8}$ \\
\hline $\mathbf{A}$ & $0 \%$ & $11 \%$ & $11 \%$ & $78 \%$ & $0 \%$ \\
\hline $\mathbf{B}$ & $6 \%$ & $60 \%$ & $28 \%$ & $6 \%$ & $0 \%$ \\
\hline $\mathbf{C}$ & $0 \%$ & $17 \%$ & $66 \%$ & $17 \%$ & $0 \%$ \\
\hline
\end{tabular}

Tabela 6 - Resultados para a métrica satisfação.

\section{$5.2 \quad$ Números Nebulosos}

Os cálculos necessários para análise usando a matemática nebulosa a partir dos resultados das métricas foram realizados utilizando o software matemático MatLab, que gerou os resultados graficamente para cada métrica estudada.

O gráfico apresentado para cada métrica representa dois conjuntos. O primeiro conjunto, simbolizado por uma linha com marcadores em forma de quadrado, representa a média dos números nebulosos (NN) obtidos para cada questão que compõem a métrica. O segundo conjunto, simbolizado pela linha com marcadores em forma de asterisco, representa o número nebuloso (NN) na forma triangular mais semelhante ao primeiro conjunto, que representa o resultado final para a métrica avaliada.

A Moda de esse número triangular nebuloso final determina o resultado final para usabilidade, conforme a escala: 0 - Muito Baixa; 2 - Baixa; 4 Média; 6 - Alta; 8 - Muito Alta.

A Amplitude de esse número triangular nebuloso final determina a dispersão média das opiniões dos usuários, e por conseqüência sua qualidade no resultado final. A amplitude está associada à confiança que se tem no valor da função de pertinência. Quanto menor a amplitude do intervalo, maior a confiança nos dados e quanto maior a amplitude do intervalo, menor a confiança que se tem nos dados [2].

Para efeito dessa metodologia, a amplitude representa a dispersão das opiniões dos usuários e é interpretada da seguinte forma: 1 - Mínima; 2 Pequena; 3 - Média; 4 - Alta.

\section{a) Facilidade de aprender}

O número triangular nebuloso obtido para medir a facilidade de aprender está apresentado na figura 1. Observa-se que a opinião média é 4 (satisfação média) com amplitude 4, indicando uma dispersão alta na opinião dos entrevistados. Resultado bastante indefinido, devendo-se aumentar a amostra e refazer os cálculos para se atingir maior confiabilidade no resultado.

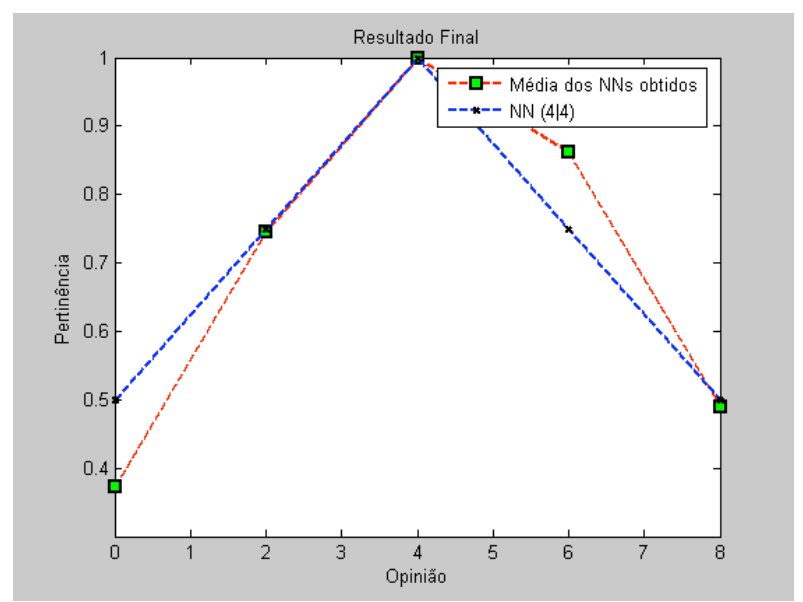

Figura 1 - Gráfico do resultado final para a métrica facilidade de aprender.

\section{b) Facilidade de relembrar}

O número triangular nebuloso obtido para medir a facilidade de aprender está apresentado na figura 2. Observa-se que a opinião média é 6 (satisfação boa) com amplitude 3 , indicando uma dispersão média na opinião dos entrevistados.

Pode-se afirmar que o software está adequado em relação à facilidade de relembrar. Mesmo com esse resultado, deve-se aumentar o tamanho da amostra com a finalidade de diminuir a amplitude do resultado. 


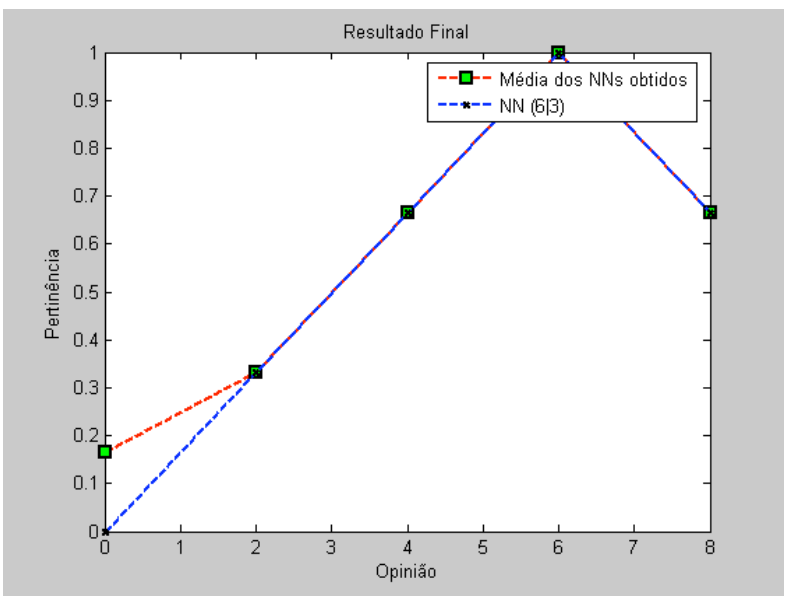

Figura 2 - Gráfico do resultado final para a métrica facilidade de relembrar.

\section{c) Controle de Erros}

O número triangular nebuloso obtido para medir o controle de erros está apresentado na figura 3. Observa-se que a opinião média é 4 (satisfação média) com amplitude 3, indicando uma dispersão média na opinião dos entrevistados.

Pode-se afirmar que o software está moderadamente adequado em relação ao controle de erros. Mesmo com esse resultado, deve-se aumentar o tamanho da amostra com a finalidade de diminuir a amplitude do resultado.

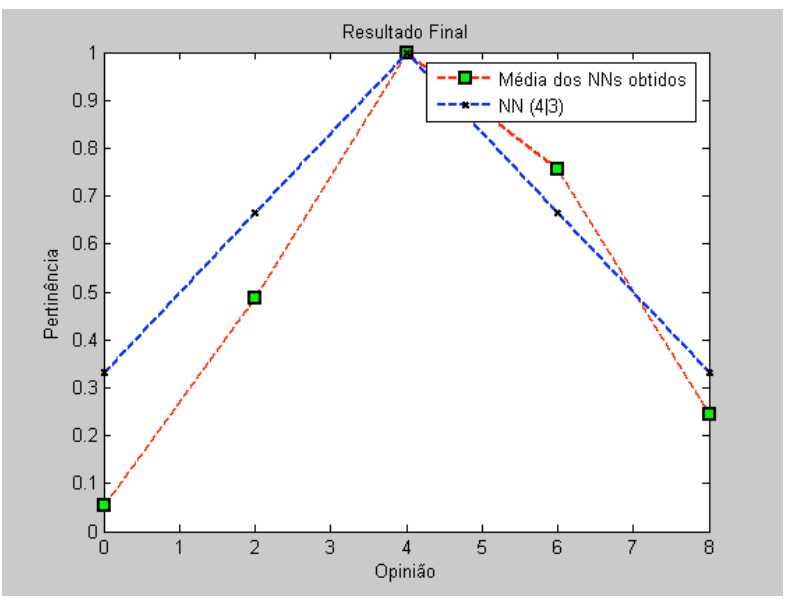

Figura 3 - Gráfico do resultado final para a métrica controle de erros.

\section{d) Eficiência}

O número triangular nebuloso obtido para medir a facilidade de aprender está apresentado na figura 4. Observa-se que a opinião média é 4 (satisfação média) com amplitude 3 , indicando uma dispersão média na opinião dos entrevistados.

Pode-se afirmar que o software está moderadamente adequado em relação à eficiência. Mesmo com esse resultado, deve-se aumentar o tamanho da amostra com a finalidade de diminuir a amplitude do resultado.

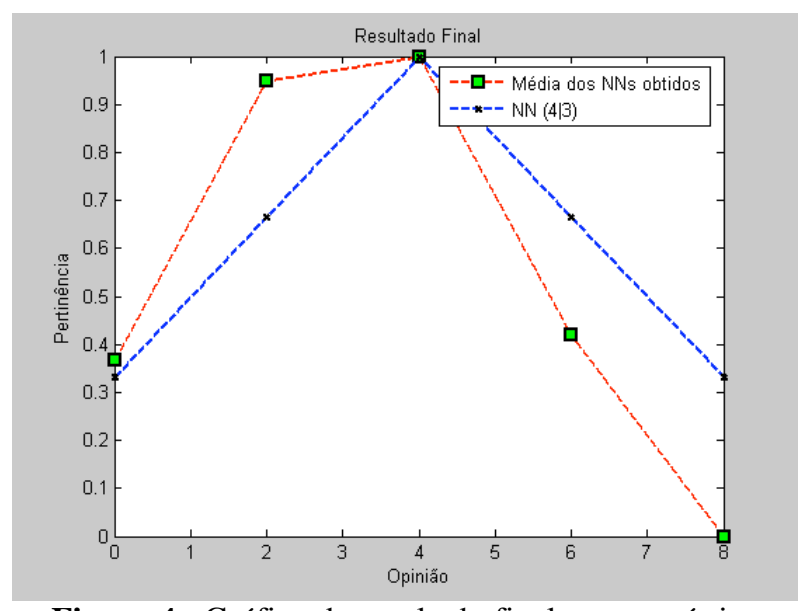

Figura 4 - Gráfico do resultado final para a métrica eficiência.

\section{e) Eficácia}

O número triangular nebuloso obtido para medir a facilidade de aprender está apresentado na figura 5. Observa-se que a opinião média é 6 (satisfação boa) com amplitude 4, indicando uma dispersão alta na opinião dos entrevistados. Pode-se afirmar que o software está adequado em relação à eficácia. Mesmo com esse resultado, deve-se aumentar o tamanho da amostra com a finalidade de diminuir a amplitude do resultado.

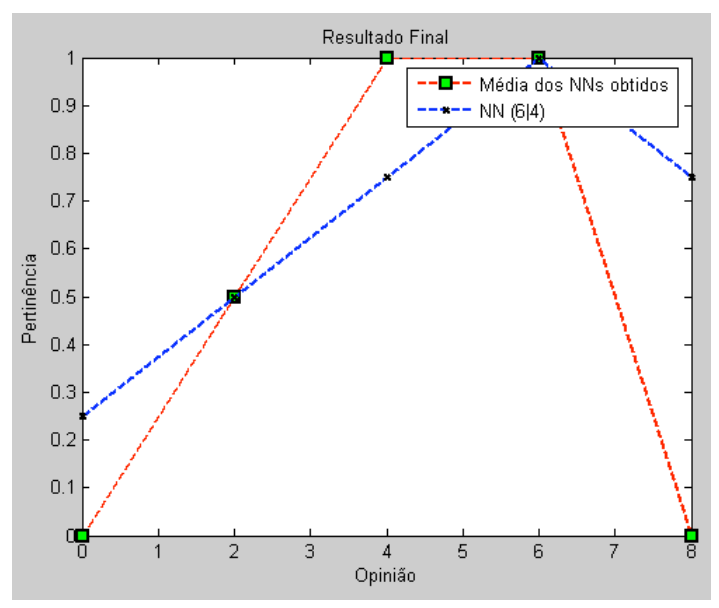

Figura 5 - Gráfico do resultado final para a métrica eficácia.

\section{f) Satisfação}

O número triangular nebuloso obtido para medir a facilidade de aprender está apresentado na figura 6. Observa-se que a opinião média é 6 (satisfação boa) com amplitude 4, indicando uma dispersão alta na opinião dos entrevistados. Pode-se afirmar que o software está adequado em relação à satisfação. Mesmo com esse resultado, deve-se aumentar o tamanho da amostra com a finalidade de diminuir a amplitude do resultado. 


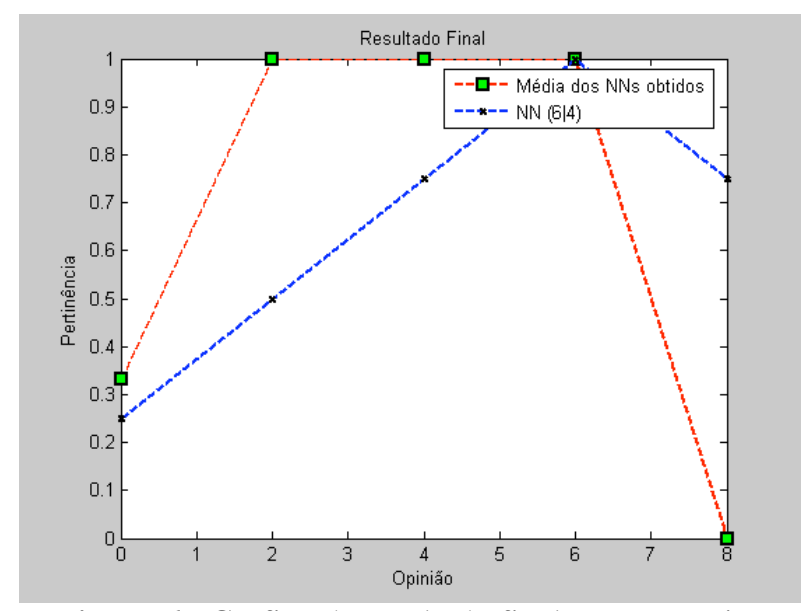

Figura 6 - Gráfico do resultado final para a métrica satisfação.

\section{Conclusões}

Os resultados obtidos com esse estudo visam apresentar uma nova vertente para avaliação de usabilidade de softwares. Dependendo do sistema analisado, esse resultado pode ser interpretado como retenção de clientes, aumento do lucro, aumento da produtividade, satisfação dos empregados, entre outros benefícios.

Nesse caso, a metodologia foi aplicada a um pacote estatístico sobre uma amostra de estudantes-usuários, ou seja, se o usuário é um iniciante no estudo da estatística, a interface do sistema não pode ser um entrave na utilização de suas funções. O pacote deve ser fácil de aprender e de relembrar, não provocar erros e concluir suas tarefas com eficiência e eficácia. Todos esses fatores implicam na satisfação final do usuário para com o produto.

Após a análise dos resultados parciais dessa pesquisa, obteve-se uma avaliação da usabilidade do pacote estatístico PHStat, conforme apresentada a seguir:

- Os usuários apresentaram facilidade média em aprender o uso do PHStat ;

- Os usuários apresentaram boa facilidade de relembrar o uso do PHStat quando passam um tempo sem utilizá-lo;

- Os usuários apresentaram satisfação média no controle de erros;

- Os usuários acharam que o PHStat tem eficiência média;

- Os usuários acharam que o PHStat tem boa eficácia;

- Os usuários ficaram satisfeitos com o uso do PHStat.

Com o intuito de ampliar a pesquisa, serão feitas novas coletas de opiniões através de questionários na internet para outros usuários do pacote PHStat em todo o País. Neste caso há um interesse em segmentar as amostras dos usuários por tempo de experiência na utilização do pacote para se verificar se a satisfação final aumenta ou não.

\section{Referências}

[1] BARRETO, Jorge M. Inteligência Artificial no Limiar do Século XXI. Florianópolis: Duplic, 2001.

[2] BRAGA, Mario J. F.; BARRETO, Jorge M.; MACHADO, Maria Augusta S. Conceitos da Matemática Nebulosa na Análise de Risco. Rio de Janeiro: Artes\& Rabiskus, 1995.

[3] CEZAR, Breno L., MACHADO, Maria Augusta S., OLIVEIRA JR, Hime A. Sistema de Apoio à Decisão na Concessão de Crédito Pessoal usando Lógica Fuzzy. Anais do Simpósio de Excelência em Gestão e Tecnologia (SEGET), Rio de Janeiro, 2006.

[4] COSTA, Alex da; RODRÍGUEZ, Antonio Gabriel; SIMAS, Etiene P. L.; ARAÚJO, Roberto da S. Lógica Fuzzy: Conceitos e Aplicações. Disponível em:

$<$ http://www.inf.unisinos.br/ cazella/dss/fuzzy_relat orio.pdf>. Acesso em: 04 nov. 2006.

[5] ISO 9126-1. Engenharia de software - Qualidade de produto. Parte 1: Modelo de qualidade. NBR ISO/IEC 9126-1. Rio de Janeiro: ABNT, 2003.

[6] ISO 9241. Ergonomic requirements for office work with visual display terminals (VDTs). ISO 9241. Switzerland: ISO, 1998.

[7] MATTAR, F.. Pesquisa de Marketing. São Paulo: Editora Atlas, 1997.

[8] NIELSEN, J. Usability Engineering. Boston, MA: Academic Press, 1993.

[9] NORMAN, D. A. User centered systems design. New York: Lawrence Earlbaum Associates, 1986.

[10] OLIVEIRA JR., Hime A. Lógica Difusa: Aspectos Práticos e Aplicações. Rio de Janeiro: Interciência, 1999.

[11] OLIVEIRA JR., Hime A., CALDEIRA, André M., MACHADO, Maria A. S., SOUZA, Reinaldo, TANSCHEIT, Ricardo. Inteligência Computacional Aplicada à Administração, Economia e Engenharia em Matlab. Rio de Janeiro, Thompson, 2007.

[12] PH S T A T . Dis poníve 1 e m : <http://www.prenhall.com/phstat/>. Acesso em: 06 mar. 2007.

[13] SANTOS, Rodrigo C. Desenvolvimento de uma metodologia para avaliação de usabilidade de sistemas utilizando a lógica Fuzzy baseado na ISO. Dissertação de Mestrado Profissionalizante em 
Administração, Faculdades IBMEC - Rio de Janeiro, 2007.

[14] SHACKEL, B. Ergonomics in design for usability. In: Harrison, M. D., \& Monk, A. F. People and computers: Designing for usability, In: HCI 86 Conference on People and Computer. New York: Cambridge University Press, p. 44-64, 1986.

[15] VERGARA, Sylvia C. Projetos e Relatórios de Pesquisa em Administração. 3 ed. São Paulo: Atlas, 2000. 